\title{
Electroacupuncture Against Ethanol-Induced Gastric Ulcer in Rats: Effects on Gastric Contractility and in the Levels of TNF- $\alpha$
}

\author{
Aline French de Lima Vitorino ${ }^{1 *}$, Loyane Almeida Gama ${ }^{2}$, Mariana Pirani Rocha ${ }^{2}$, Luciana Aparecida Corá ${ }^{3}$, José \\ Ricardo de Arruda Miranda ${ }^{2}$ and Madileine Francely Américo ${ }^{1}$
}

${ }^{1}$ Institute of Biological and Health Sciences, Brazil

${ }^{2}$ Institute of Biosciences, Brazil

${ }^{3}$ Centre of Health Sciences, Campus Governador Lamenha Filho, Brazil

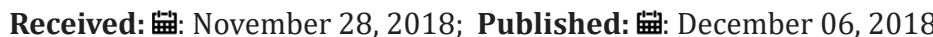

*Corresponding author: Aline French de Lima Vitorino, Madileine Francely Américo, Institute of Biological and Health Sciences, Valdon Varjão Avenue, no. 6.390. CEP: 78600-000 Barra do Garças, Mato Grosso, Brazil

\begin{abstract}
Objective: To correlate the effects of the Electroacupuncture (EA) with the levels of TNF- $\alpha$ and the gastric contractility in both ulcerated and non-ulcerated control rats.

Methods: Before the experiments, a magnetic marker was surgically implanted in the gastric serosa of all animals used in this study. Ten days after the surgical procedure, the animals were randomly assigned in groups ( $\mathrm{n}=9$ per group) whose received by gavage phosphate buffered saline (non-ulcerated control groups) or ethanol (ulcerated groups). EA treatments were applied every three days during 16 consecutive days in both control (C) and ulcerated (U) groups at sham (S) point (CS and US) and ST-36 acupuncture point (CST-36 and UST-36). Magnetic and electrical gastric activity recordings were also performed. TNF- $\alpha$ was measured by ELISA and all data were statistically analysed by ANOVA. Results: In the ulcerated animals which received sham EA (US), the frequency and amplitude of contractions were reduced, whereas the levels of TNF- $\alpha$ were increased. EA applied at UST-36 group had significantly increases in the frequency and in the amplitude of contractions. A clear inverse correlation ( $\rho=0.9)$ between motility parameters and TNF- $\alpha$ levels in ulcerated rats was observed.
\end{abstract}

Conclusion: EA in ST-36 had improves the gastric contractility in ulcerated rats by a pathway that involves decreases in the levels of TNF- $\alpha$.

Keywords: Cytokine; Electroacupuncture; Gastric Contractility; Gastric Ulcer

Abbreviations: GI: Gastrointestinal; TNF- $\alpha$ : Tumor Necrosis Factor-Alpha; EA: Electroacupuncture; EGG: Electrogastrography; ACB: Alternating Current Biosusceptometry; CS: Control Groups at Sham; US: Ulcerated Groups at Sham; FFT: Fast Fourier Transform; RSA: Running Spectrum Analysis; AUC: Area Under Contraction; R: Recordings; ANOVA: Analysis of Variance; SD: Standard Deviation; NSAID's: Non-Steroidal Anti-Inflammatory Drugs; ICC: Cells of Cajal

\section{Introduction}

Gastric ulcer is a common disease with multiple etiologist and results of an imbalance that occurs between aggressive and protective factors in the gastrointestinal (GI) mucosa [1-3]. Besides, this condition is associated with increased secretion of pro-inflammatory cytokines such as the tumor necrosis factoralpha (TNF- $\alpha$ ) $[4,5]$. At least 4 million people worldwide suffer from peptic ulcer and its complications such as GI motor disturbances, indigestion and gastroparesis (i.e., slowed gastric emptying in the absence of obstruction) affects $10-20 \%$ of the patients $[3,6,7]$. Proton pump inhibitors are the most commonly drugs prescribed for the treatment of peptic ulcer disease. Despite their safety and tolerability, the adverse effects associated with long-term use, lack of curative effects and high costs of the new drugs have renewed public interest in complementary and alternative treatments including Electroacupuncture (EA) [8,9]. Studies have shown that the needle-stimulation of Zusanli point (stomach 36, ST-36) outcomes preventive effects, which includes improvements in the immune function, decreasing the gastric acid secretion [10], and also in the levels of pro-inflammatory cytokines in the rat's serum $[11,12]$. 
In terms of motility, EA improves the gastric slow-wave rhythmicity and antral contractile activity [13]; however, there is still a lack of scientific background available to evidence on the clinical significance of EA in the treatment of peptic ulcer disease. Electrogastrography (EGG) and Alternating Current Biosusceptometry (ACB) have been proposed as innovative and minimally invasive techniques for the study of GI motor activity [14]. ACB is a magnetic technique that was previously validated to register mechanical contractility resulting of the GI wall movements generated directly by the smooth muscle $[14,15]$. EGG is employed towards monitoring gastric myoelectrical activity using cutaneous electrodes placed on the abdominal surface $[16,17]$. Both techniques in association enable to register electrical and mechanical activity simultaneously and in real time [18]. As the evidences concerning the benefits of EA in the improvement of gastric function in the peptic ulcer disease still need to be confirmed, the aim of our study was to correlate the effects of the EA with the levels of TNF- $\alpha$ and the gastric contractility in both ulcerated and non-ulcerated control rats.

\section{Methods and Methods}

\section{Animals and Surgical Procedures}

All experiments were approved by the Institutional Animal Care and Use Committee (Protocol number 23108.002959/13-3 - Federal University of Mato Grosso) and were conducted in accordance with animal ethics guidelines consistent with the National Research Council 'Guide for the Care and Use of Laboratory Animals'. Male Wistar rats (body weight 200-300g) were housed individually in polypropylene cages under controlled lighting conditions $(12 \mathrm{~h} / 12 \mathrm{~h}$ light/dark cycle) at room temperature $\left(22-26^{\circ} \mathrm{C}\right)$ with free access to water and food. After 12-hours long fasting, a laparotomy was performed in order to implant a magnetic marker. For this surgical procedure, all the animals were anesthetized with an intramuscular injection of ketamine (30 mg/ kg) and xylazine $(15 \mathrm{mg} / \mathrm{kg})$. A magnetic marker (diameter $3.5 \mathrm{~mm}$; height $3.0 \mathrm{~mm}$ ) was implanted in the seromuscular layer by suturing it in the gastric corpus, using a 6/0 thread, $3 \mathrm{~cm}$ far from the pylorus [18]. The recovery period for all animals lasting up to 10 days and they were acclimated to restraint by the same researcher before the experiments.

\section{Induction of Gastric Ulcer and Electroacupuncture Treatment}

Ten days after the surgical procedure, the animals were randomly assigned in four groups ( $\mathrm{n}=9$ per group): ulcerated and treated to EA at ST-36 acupuncture point (UST-36); ulcerated and treated to EA at sham point (US); control and treated to EA at ST36 acupuncture point (CST-36); control and treated to EA at sham point (CS). Gastric ulcer was induced in 18 animals (ulcerated groups) with $1 \mathrm{~mL}$ of absolute ethanol orally administered. For the control groups, animals $(n=18)$ received $1 \mathrm{~mL}$ of phosphate buffered saline (PBS) by the same route [19]. EA treatments were applied every three days during 16 consecutive days in both control (C) and ulcerated (U) groups at sham (S) point (CS and US) or at ST36 acupuncture point (CST-36 and UST-36) [13]. ST-36 acupuncture point was located at the anterior tibial muscle, $5 \mathrm{~mm}$ lateral to and below the anterior tubercle of the tibia [11]. Two stainless-steel needles $(0.25 \times 15 \mathrm{~mm}$, DongBang Acupuncture, Inc., Chungnam, Korea) were inserted bilaterally $(0.5-1 \mathrm{~mm}$ deep) in the ST36 acupuncture point and in sham point. Sham point was located at 5 mm posteriorly and inferiorly to the point ST-36 based on needling off point and was confirmed by absence of electrical detection. The anode and cathode lead from an electrical stimulator (WQ IOD1; Donghua, China) were connected to the two acupuncture needles. A direct current with an intensity range of $4 \mathrm{~mA}$ and stimulation frequency of $15 \mathrm{~Hz}$ was applied during $20 \mathrm{~min}$.

\section{ACB and EGG Techniques}

ACB sensors consist of assemble of induction coils composed of an excitation coil (outer) and a detection coil (inner) in a firstorder gradiometric configuration. Details on the instrumentation were described elsewhere and the sensor has been improved for laboratory animal studies [14]. Briefly, the magnetic signals detected by the sensor are derived from the magnetic marker implanted in the gastric serosa [15]. As the signal detection depends on the distance between the sensor and the magnetic material, the signal recorded by the sensor is modulated by the changes in the position of the marker during the contraction and relaxation of the gastric wall. Electrogastrography (EGG) provides non-invasive monitoring of gastric myoelectrical activity through cutaneous electrodes placed on the abdominal skin over the stomach.

\section{Gastric Activity Recordings}

Simultaneous recordings of both mechanical (ACB) and electrical (EGG) activities were performed as follows. After the ingestion of 2 $\mathrm{g}$ of standard chow, animals were anesthetized by intraperitoneal injection of pentobarbital sodium $(30 \mathrm{mg} / \mathrm{kg}$, Abbott Laboratories, Chicago, USA) and they were kept supine during the 40-minute recordings period. The magnetic sensor (Br4Science $₫$, São Paulo, Brazil) was placed on the anterior surface of the abdomen and a continuous ACB signal recording was acquired. For EGG recordings, surface electrodes in a bipolar configuration were placed near to the ACB sensor in the abdomen and were connected to the BIOPAC amplifier system. Simultaneous signals were acquired at a $20 \mathrm{~Hz}$ / channel rate digitized using a multi-channel recorder (MP100 System; BIOPAC Inc., Santa Barbara, USA) and stored for further analyses. ACB and EGG recordings (R) were performed three times (Figure 1): R1, at the first day before the EA treatment; R2, at the seven day, after the third EA treatment; and R3, at the sixteenth day, after the sixth EA treatment. In addition, in order to verify the influence of EA treatment on the recovery of the animals during the experiments, weight gain percentage (\%) was determined from the first to the third recording session. 


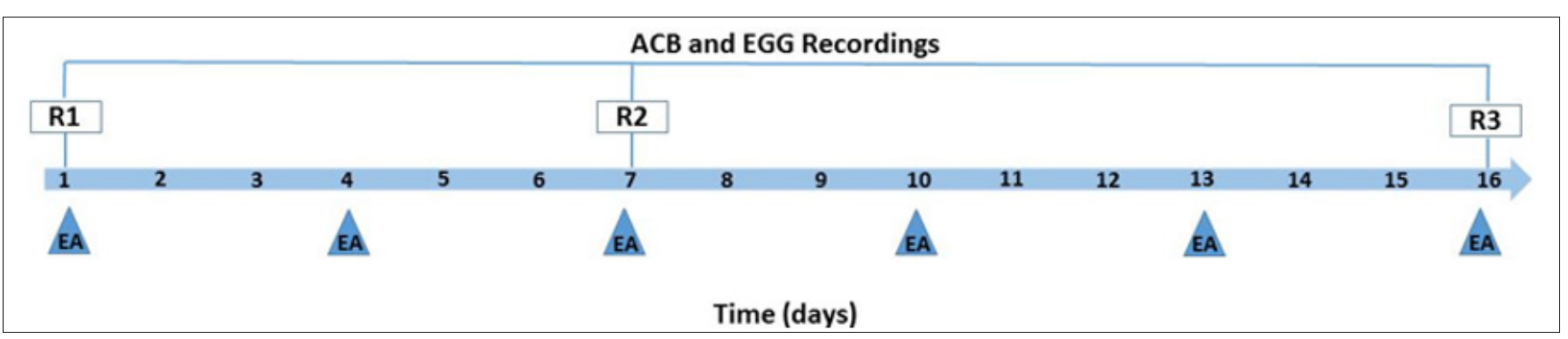

Figure 1: Schematic timeline for ACB and EGG recordings and for EA treatment scheduled for the animals.

\section{Immunological Analysis}

At the end of the R3 measurements, all the animals were killed by decapitation under overdose anaesthesia towards collecting gastric tissue sample. TNF- $\alpha$ (product number 558535) was quantified in the gastric tissue by ELISA according to manufacturer's instructions (R\&D Systems, Minneapolis, USA) in order to detect cytokine with a minimum detection limit of $10 \mathrm{pg}$./mL. Values were expressed as cytokine picograms per millilitre (pg./ml).

\section{Data Analysis}

Gastric activity recordings were initially analysed in MATLAB (MathWorks, Inc., Natick, USA) by visual inspection and thereafter using bi-directional Butterworth band-pass filters by Fast Fourier Transform (FFT) and by Running Spectrum Analysis (RSA). The signals were also analysed using band-pass filters with a cut off frequency of $0.03-0.15 \mathrm{~Hz}(1.8-9.0 \mathrm{cpm}$ - cycles per minute) to determine the frequency of contractions. To determine the amplitude of contractions, area under contraction (AUC) was calculated using the same filters described above. The normality of continuous variables was tested using the Kolmogorov and Smirnov's test. For variables normally distributed, data were analysed using the oneway analysis of variance (ANOVA) followed by the Tukey's multiple comparisons test. Pearson correlation coefficient was adopted to assess the relationship between variables. A value of $\mathrm{p}<0.05$ was considered significant. Data were expressed as mean \pm standard deviation (SD).

\section{Results}

Gastric activity signals registered by ACB in the ulcerated rat before and after the EA treatment are shown in the Figure 2. In comparison with EA at ST-36 acupuncture point, it was identified irregular patterns of amplitude and frequency in ulcerated rat before EA treatment. The frequency and amplitude of gastric contractions recorded for all groups during EA protocol is showed in Table 1. A significant decrease in the frequency of contraction in the ulcerated rats was observed $(\mathrm{p}<0.01)$. Moreover, the UST36 rats showed increases in the frequency of contraction similar to that observed in the control group, but significantly higher than that observed in the US group $(\mathrm{p}<0.001)$. Our results also indicate that the effects of EA on the frequency of contraction for CST-36 animals were significantly reduced when compared with those observed for UST-36 group. The amplitude of contractions tends to be lower in the ulcerated non-treated rats. However, in the UST36 group it was observed a significant increase in the amplitude of contraction compared with non-treated groups.

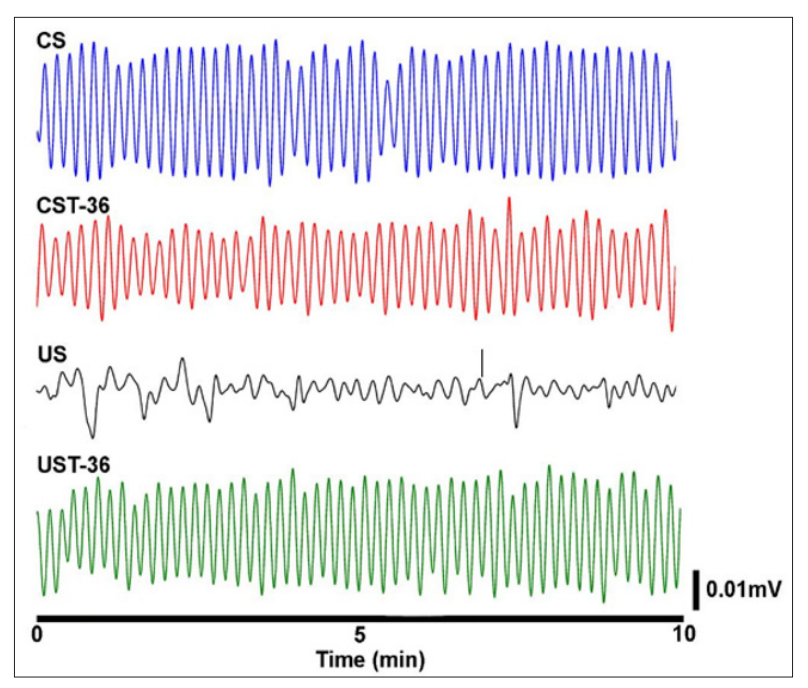

Figure 2: Typical ACB contractility recordings obtained for control (A) and for ulcerated (B) groups. The signals were acquired before and after EA treatment in the control group (CS and CST-36) and in the ulcerated group (US and UST-36). 
Table 1: Frequency (cpm) and Amplitude (mv.s) of Gastric Contractions Obtained by ACB and EGG Techniques during the Recordings at R1, R2 and R3 Protocols for All Control and Treated Groups.

\begin{tabular}{|c|c|c|c|c|c|c|c|c|}
\hline \multicolumn{9}{|c|}{$\begin{array}{c}\text { Frequency (cpm) and Amplitude (mv.s) of Gastric Contractions Obtained by ACB and EGG Techniques during the Recordings at R1, R2 and R3 } \\
\text { Protocols for All Control and Treated Groups }\end{array}$} \\
\hline & CS & CST36 & CS vs CST36 & US & UST36 & CS vs US & CST36 vs US & CST36 vs US \\
\hline \multicolumn{9}{|c|}{ Frequency } \\
\hline \multicolumn{9}{|c|}{ ACB } \\
\hline R1 & $5.04 \pm 0.24$ & $4.80 \pm 0.26$ & & $4.50 \pm 0.35$ & $5.28 \pm 0.39$ & $\mathrm{P}<0.01$ & $\mathrm{P}<0.05$ & -- \\
\hline $\mathrm{R} 2$ & $5.20 \pm 0.29$ & $4.92 \pm 0.59$ & -- & $4.57 \pm 0.19$ & $5.33 \pm 0.28$ & $\mathrm{P}<0.01$ & $\mathrm{P}<0.05$ & -- \\
\hline R3 & $5.01 \pm 0.35$ & $5.01 \pm 0.26$ & -- & $4.24 \pm 0.47$ & $5.12 \pm 0.21$ & $\mathrm{P}<0.001$ & $\mathrm{P}<0.01$ & $\mathrm{P}<0.01$ \\
\hline \multicolumn{9}{|c|}{ EGG } \\
\hline R1 & $5.04 \pm 0.39$ & $4.96 \pm 0.32$ & -- & $4.11 \pm 0.38$ & $5.12 \pm 0.40$ & $\mathrm{P}<0.01$ & $\mathrm{P}<0.05$ & $\mathrm{P}<0.05$ \\
\hline R2 & $5.15 \pm 0.48$ & $5.13 \pm 0.20$ & -- & $4.18 \pm 0.58$ & $5.36 \pm 0.85$ & $\mathrm{P}<0.001$ & $\mathrm{P}<0.05$ & $\mathrm{P}<0.05$ \\
\hline R3 & $4.89 \pm 0.32$ & $5.17 \pm 0.43$ & -- & $3.98 \pm 0.60$ & $5.15 \pm 0.57$ & $\mathrm{P}<0.001$ & $\mathrm{P}<0.05$ & $\mathrm{P}<0.001$ \\
\hline \multicolumn{9}{|c|}{ Amplitude } \\
\hline \multicolumn{9}{|c|}{ ACB } \\
\hline R1 & $0.55 \pm 0.18$ & $0.54 \pm 0.19$ & -- & $0.21 \pm 0.05$ & $0.68 \pm 0.19$ & $\mathrm{P}<0.01$ & -- & -- \\
\hline R2 & $0.58 \pm 0.25$ & $0.56 \pm 0.22$ & -- & $0.30 \pm 0.08$ & $0.84 \pm 0.52$ & $\mathrm{P}<0.001$ & -- & -- \\
\hline \multirow[t]{2}{*}{ R3 } & $0.54 \pm 0.15$ & $0.49 \pm 0.22$ & -- & $0.30 \pm 0.13$ & $0.89 \pm 0.23$ & $\mathrm{P}<0.001$ & -- & $\mathrm{P}<0.05$ \\
\hline & & & & EGG & & & & \\
\hline $\mathrm{R} 1$ & $2.10 \pm 0.84$ & $1.73 \pm 0.60$ & -- & $1.75 \pm 0.28$ & $2.24 \pm 0.98$ & $\mathrm{P}<0.05$ & -- & -- \\
\hline $\mathrm{R} 2$ & $1.97 \pm 0.92$ & $1.75 \pm 0.57$ & -- & $1.50 \pm 0.58$ & $2.42 \pm 1.21$ & $\mathrm{P}<0.05$ & -- & -- \\
\hline R3 & $1.91 \pm 1.03$ & $1.64 \pm 0.62$ & -- & $1.44 \pm 1.03$ & $2.61 \pm 1.06$ & $\mathrm{P}<0.05$ & -- & -- \\
\hline
\end{tabular}

Data are expressed as mean \pm SD ( $n=9$ per group). CS: Control Sham, CST-36: Control ST- 36, US: Ulcer Sham, UST-36: Ulcer ST-36.

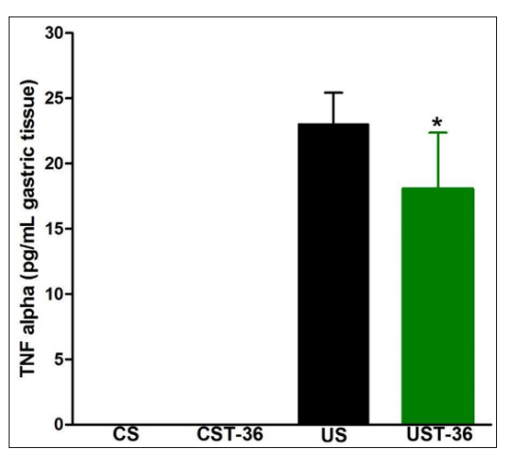

Figure 3: Levels of TNF-a obtained in the gastric tissue of rats enrolled in all groups analysed. ${ }^{*} \mathrm{p}<0.05$, compared to US.

Weight gain along the experiments was significantly increased in the ulcerated rats treated with EA compared with ulcerated rats without treatment $(21.2 \pm 2.9 \%$ and $12.0 \pm 3.3 \%$, respectively $)$ $(\mathrm{p}<0.05)$. For control group, weight gain was not affected by EA treatment $(18.0 \pm 3.3 \%$ after treatment and $22.5 \pm 3.2 \%$ without treatment). Figure 3 shows the TNF- $\alpha$ levels obtained in the gastric tissue for all animal's groups. In ulcerated rats the levels of TNF- $\alpha$ were increased in comparison with non-ulcerated control animals. However, a significant decrease in the TNF- $\alpha$ levels was obtained in UST-36 group compared with the US group. The correlation with TNF- $\alpha$ and contractility parameters (frequency and amplitude of contractions) for all groups is shown in Table 2. There is no correlation between TNF- $\alpha$ levels and contractility parameters for untreated control groups (CS). Interestingly, a strong inverse correlation with contractility parameters and TNF- $\alpha$ levels was obtained for ulcerated rats (US). This negative correlation is maintained even with treatment in ulcerated animals (UST-36) and a positive correlation between TNF- $\alpha$ levels and frequency of contractions registered by ACB in CST-36 group was also found.

Table 2: Correlation between TNF-A Levels with Motility Parameters Recorded from All Groups by ACB and EGG.

\begin{tabular}{|c|c|c|c|c|c|c|c|c|}
\hline \multicolumn{9}{|c|}{ Correlation between TNF-A Levels with Motility Parameters Recorded from All Groups by ACB and EGG } \\
\hline & \multicolumn{2}{|c|}{ CS } & \multicolumn{2}{|c|}{ CST-36 } & \multicolumn{2}{|c|}{ US } & \multicolumn{2}{|c|}{ UST-36 } \\
\hline & $\rho$ & $\mathbf{P}$ & $\rho$ & $\mathbf{P}$ & $\rho$ & $\mathbf{P}$ & $\rho$ & $\mathbf{P}$ \\
\hline \multicolumn{9}{|c|}{ Frequency } \\
\hline $\mathrm{ACB}$ & -0.34 & 0.3639 & 0.86 & 0.0026 & -0.90 & 0.0009 & -0.82 & 0.0079 \\
\hline EGG & -0.55 & 0.1231 & 0.26 & 0.4984 & -0.90 & 0.0009 & -0.81 & 0.0083 \\
\hline \multicolumn{9}{|c|}{ Amplitude } \\
\hline
\end{tabular}

Cite this article: Aline French de Lima V, Loyane Almeida G, Mariana Pirani R, Luciana Aparecida C, et al. Electroacupuncture Against Ethanol-Induced Gastric Ulcer in Rats: Effects on Gastric Contractility and in the Levels of TNF- $\alpha$. Biomed J Sci \& Tech Res 11(5)-2018. BJSTR. MS.ID.002153. DOI: 10.26717/ BJSTR.2018.11.002153. 


\begin{tabular}{|l|l|l|l|l|l|l|l|}
\hline ACB & 0.07 & 0.8647 & 0.45 & 0.2242 & -0.92 & 0.0005 & -0.90 \\
\hline EGG & -0.17 & 0.6682 & 0.28 & 0.4841 & -0.25 & 0.5000 & -0.10 \\
\hline
\end{tabular}

Data are presented as mean \pm SD (n=9). CS: Control Sham, CST-36: Control ST-36, US: Ulcer Sham, UST-36: Ulcer ST-36.

\section{Discussion}

Our results suggest that EA in ST-36 acupuncture point was able to improve the gastric contractility in ulcerated animals by a pathway that seems to involve decreases in the TNF- $\alpha$ levels obtained in the gastric tissues. EA also improved significantly the weight gain in the ulcerated animals $(\mathrm{p}<0.05)$. Besides, a strong inverse correlation with TNF- $\alpha$ levels and gastric contractility was obtained for ulcerated rats. Otherwise, EA applied to the control animals showed positive correlation with TNF- $\alpha$ and the frequency of contractions. The differences observed in this study reinforces that acupuncture points are pathophysiologically dynamic entities connected with homeostasis fluctuations, which contributes to the improvements from the baseline [20,21]. The effects of acupuncture treatment on the gastric contractility in ulcerated animals are still unclear. In general, gastric mucosa injuries induced by ethanol and non-steroidal anti-inflammatory drugs (NSAIDs) are linked to increases in the gastric motility [22,23]. However, the gastric motor patterns depending on the model of ulcer induction. The ethanol exposure seems to inhibit both slow wave activity and small intestinal motor activity in rats $[24,25]$. Our data corroborate those previous studies, showing that in ulcerated animals the frequency and amplitude of gastric contractions were smallest than those obtained in non-ulcerated control rats (Table 1).

The bradygastry observed in ulcerated animals can be associated to several symptoms of peptic ulcer such as pain, indigestion, early satiety, loss of appetite and vomiting $[3,26]$. The acupuncture treatment seems to be able to normalize the acid secretion and GI motility with improvements in the visceral pain $[27,28]$. Studies have shown that EA at ST36 point can improve the blood flow in gastric mucosa. EA at ST36 also regulates the gastric motility by rising frequency and amplitude of contractions in rats [25]. Moreover, this acupuncture point is often associated with the recovery of the damaged networks of Interstitial Cells of Cajal (ICC) and pacemakers towards propagating slow waves in the stomach of rats [29]. In our study, for the UST-36 rats the frequency of contractions was similar to that observed in non-ulcerated controls (CST-36) with a significant increase when compared with US group $(\mathrm{P}<0.05)$. The amplitude of contractions was lower for the US animals during all experiments. On the other hand, higher amplitude values for the UST-36 rats compared with US $(\mathrm{P}<0.05)$ was obtained. Ethanol-induced gastric ulcer in experimental animals plays an important role towards understanding the mechanisms of mucosal injury and secretion of pro-inflammatory cytokines such as TNF- $\alpha$, Interleukin (IL)-1 $\beta$ and IL-6 $[22,30]$. Gastric ulcer induced by ethanol in rats increased the level of TNF- $\alpha$ in gastric tissue compared with control groups (Figure 3).

It is known that the production of pro-inflammatory cytokines such as IL- 6 and TNF- $\alpha$ is notably increased in the serum and in the gastric tissue of the ethanol-induced ulcer model $[31,32]$. As an inflammatory cytokine with pleiotropic functions, TNF- $\alpha$ is associated with the induction and progression of inflammatory disorders [32]. These pro-inflammatory mediators are regulated by nuclear factor kappa B (NF-KB) signaling pathway [2], one of the most widely recognized intracellular signaling pathway in inflammatory responses [32]. Evidences suggest that the inhibition of NF-kB activity may lead to reduce the severity of inflammatory diseases [33]. Under inflammation conditions, studies also showed that decreases in the levels of TNF- $\alpha$ after EA $[11,34]$ is associated with anti-inflammatory effects [35]. Our data suggest that EA treatment had an effective effect in the reduction of the inflammatory process, since the levels of TNF- $\alpha$ in the gastric tissue was decreased. Our study also revealed that the increase in the TNF- $\alpha$ levels led to impairment of the gastric motility parameters (Table 2). However, after the treatment with EA at ST-36 acupuncture point, there was observed an improvement in gastric motor function. These data suggest that the effect of EA may be mediated, at least in part, by the inhibition of the expression of TNF- $\alpha$ and by other unknown mechanisms. This fact becomes more evident considering that the CST-36 group also had the motor function altered. Our data showed that the correlation with TNF- $\alpha$ obtained in gastric tissue and the mechanical recordings (ACB) was more consistent than that observed with electrical (EGG) recordings.

As the interpretation of electrical findings can be rather controversial [36], and their relationship with mechanical activities is still difficult to understand [37], the use of EGG and ACB techniques simultaneously can contribute to enhancing the knowledge about motility issues $[14,16]$. This approach allowed us inferring that the recovery of the gastric tissue after EA treatment led to further improvement in motility parameters. Potential weaknesses of our study were the multiple handling of the animal and the effects of stress [38]. However, some approaches were adopted towards minimizing predictable interferences. Firstly, the animals were trained by the same researcher and they were handled gently several times a day in order to reduce the impact of stress on the experimental data [39]. Several clinical and laboratory studies had been conducted in order to clarify the mechanisms behind systemic effects of EA treatment on the GI motility. However, such effects rely on several variables including species, acupuncture procedures, different signaling pathways and, mainly, initial motor activity [40]. In summary, our study showed that EA treatment improves the gastric contractility of ulcerated rats and decreases proinflammatory cytokine TNF- $\alpha$ levels in gastric tissue. As the exact mechanism that explains how EA treatment acts is still unclear, further studies are needed in order to investigate complimentary pathways and critical factors associated to the electroacupuncture to explain those outcomes.

\section{Acknowledgement}

The authors are grateful to Dr. Anderson Sá-Nunes from Institute of Biomedical Sciences, University of Sao Paulo, for helping with the ELISA technique and for the critical reading of the manuscript. The 
authors are thankful to Andrieli Taise Hauschildt and Luana dos Anjos Ramos for their technical assistance. The authors are also grateful to Department of Immunology, ICB/USP for their valuable contribution in study design and immunological analysis.

\section{Authorship Contribution}

Madileine Francely Américo, Luciana Aparecida Corá and José Ricardo de Arruda Miranda conceived and designed the study. Aline French de Lima Vitorino and Loyane Almeida Gama performed the experiments. Madileine Francely Américo, Luciana Aparecida Corá, Aline French de Lima Vitorino, Mariana Pirani Rocha and Loyane Almeida Gama performed the data analyses. All authors wrote, read and approved the final manuscript.

\section{Financial Support}

This study was financed in part by the Coordenação de Aperfeiçoamento de Pessoas de Nível Superior - Brasil (CAPES) Finance Code 001.

\section{References}

1. Yeomans ND, Naesdal J (2008) Systematic review: ulcer definition in NSAID ulcer prevention trials. Aliment Pharmacol Ther 27(6): 465-472.

2. Li WF, Hao DJ, Fan T, Huang HM, Yao H, et al. (2014) Protective effect of chelerythrine against ethanol-induced gastric ulcer in mice. Chem Biol Interact 208: 18-27.

3. Ramakrishnan K, Salinas RC (2007) Peptic Ulcer Disease. Am Fam Physician 76(7): 1005-1012.

4. Sugimoto M, Yamaoka Y, Furuta T (2010) Influence of interleukin polymorphisms on development of gastric cancer and peptic ulcer. World J Gastroenterol 16(10): 1188-1200.

5. Shimizu N, Watanabe T, Arakawa T, Fujiwara Y, Higuchi K, et al. (2000) Pentoxifylline accelerates gastric ulcer healing in rats: roles of tumor necrosis factor alpha and neutrophils during the early phase of ulcer healing. Digestion 61(3): 157-164.

6. Thorsen K, Søreide JA, Kvaløy JT, Glomsaker T, Søreide K (2013) Epidemiology of perforated peptic ulcer: Age- and gender-adjusted analysis of incidence and mortality. World J Gastroenterol 19(3): 347354.

7. Kanaizumi T, Nakano H, Matsui T, Tatsumi H, Ishikawa H, et al. (1989) Gastric emptying in patients with gastric and duodenal ulcer. Tohoku J Exp Med 158(2): 133-140.

8. Chubineh S, Birk J (2012) Proton Pump Inhibitors: The Good, the Bad, and the Unwanted. South Med J 105(11): 613-618.

9. Patwardhan B, Warude D, Pushpangadan P, Bhatt N (2005) Ayurveda and traditional chinese medicine: A comparative overview. Evid Based Complement Alternat Med 2(4): 465-473.

10. Chang SL, Tsai CC, Lin JG, Hsieh CL, Lin RT, et al. (2005) Involvement of serotonin in the hypoglycemic response to $2 \mathrm{~Hz}$ electroacupuncture of zusanli acupuncture point (ST36) in rats. Neurosci Lett 379(1): 69-73.

11. Wang WJ, Lu J, Niu CS, Huang YR, Ma Q et al. (2010) Effects of electroacupuncture of unilateral and bilateral "zusanli" (ST 36) on serum TNF-alpha, IL-1 and IL-4 levels in rats with chronic inflammatory pain. Zhen Ci Yan Jiu 35(6): 429-432.

12. Torres-Rosas R, Yehia G, Peña G, Mishra P, del Rocio Thompson-Bonilla $\mathrm{M}$, et al. (2014) Dopamine mediates vagal modulation of the immune system by electroacupuncture. Nat Med 20(3): 291-295.

13. Ouyang H, Yin J, Wang Z, Pasricha PJ, Chen JD (2002) Electroacupuncture accelerates gastric emptying in association with changes in vagal activity. Am J Physiol Gastrointest Liver Physiol 282(2): 390-396.
14. Américo MF, Marques RG, Zandoná EA, Andreis U, Stelzer M, et al. (2010) Validation of ACB in vitro and in vivo as a biomagnetic method for measuring stomach contraction. J Neurogastroenterol Motil 22(12): 1340-1344

15. Quini CC, Américo MF, Corá LA, Calabresi MF, Alvarez M, et al. (2012) Employment of a noninvasive magnetic method in the evaluation of gastrointestinal transit in rats. J Biol Eng 6: 6.

16. Yin J, Chen JD (2013) Electrogastrography: Methodology, Validation and Applications. J Neurogastroenterol Motil 19(1): 5-17.

17. Parkman HP, Hasler WL, Barnett JL, Eaker EY, American Motility Society Clinical GI Motility Testing Task Force (2003) Eletrogastrography: a document prepared by the gastric section of the American Motility Society Clinical GI Motility Testing Task Force. J Neurogastroenterol Motil 15(2): 89-102.

18. Marques RG, Américo MF, Spadella CT, Corá LA, Oliveira RB, et al. (2014) Different patterns between mechanical and electrical activities: an approach to investigate gastric motility in a model of long-term diabetic rats. Physiol Meas 35(1): 69-81.

19. Santos RC, Kushima H, Rodrigues CM, Sannomiya M, Rocha LR, et al. (2012) Byrsonima intermedia A. Juss.: gastric and duodenal anti-ulcer, antimicrobial and antidiarrheal effects in experimental rodent models. J Ethnopharmacol 140(2): 203-212.

20. Zhou W, Benharash P (2014) Effects and mechanisms of acupuncture based on the principle of meridians. J Acupunct Meridian Stud 7(4): 190193.

21. Cheng KJ (2014) Neurobiological mechanisms of acupuncture for some common illnesses: a clinician's perspective. J Acupunct Meridian Stud 7(3): 105-114.

22. 22. Diniz LR, Vieira CF, Santos EC, Lima GC, Aragão KK, et al. (2013) Gastroprotective effects of the essential oil of Hyptis crenata Pohl ex Benth. on gastric ulcer models. J Ethnopharmacol 149(3): 694-700.

23. Takeuchi K (2012) Pathogenesis of NSAID-induced gastric damage: importance of cyclooxygenase inhibition and gastric hypermotility. World J Gastroenterol 18(18): 2147-2160.

24. Subramanya SB, Stephen B, Nair SS, Schäfer KH, Lammers WJ (2015) Effect of Ethanol exposure on slow wave activity and smooth muscle contraction in the rat small intestine. Dig Dis Sci 60(12): 3579-3589.

25. Lin YP, Yi SX, Yan J, Chang XR (2007) Effect of acupuncture at FootYangming Meridian on gastric mucosal blood flow, gastric motility and brain-gut peptide. World J Gastroenterol 13(15): 2229-2233.

26. Malfertheiner P, Chan FK, McColl KE (2009) Peptic ulcer disease. Lancet 374(9699): 1449-1461.

27. Takahashi $\mathrm{T}$ (2006) Acupuncture for functional gastrointestinal disorders. J Gastroenterol 41(5): 408-417.

28. Ouyang H, Chen JD (2004) Therapeutic roles of acupuncture in functional gastrointestinal disorders. Aliment Pharmacol Ther 20(8): 831-841.

29. Chen Y, Xu JJ, Liu S, Hou XH (2013) Electroacupuncture at ST36 ameliorates gastric emptying and rescues networks of interstitial cells of Cajal in the stomach of diabetic rats. PloS One 8(12): e83904.

30. Kwiecień S, Brzozowski T, Konturek SJ (2002) Effects of reactive oxygen species action on gastric mucosa in various models of mucosal injury. J Physiol Pharmacol 53(10): 39-50.

31. Mei X, Xu D, Xu S, Zheng Y, Xu S (2012) Novel role of Zn (II)-curcumin in enhancing cell proliferation and adjusting proinflammatory cytokinemediated oxidative damage of ethanol-induced acute gastric ulcers. Chem Biol Interact 197(1): 31-39.

32. Zhao Z, Gong S, Wang S, Ma C (2015) Effect and mechanism of evodiamine against ethanol-induced gastric ulcer in mice by suppressing Rho/NF-кB pathway. Int Immunopharmacol 28(1): 588-595. 
33. Atreya I, Atreya R, Neurath MF (2008) NF- $\mathrm{B}$ in inflammatory bowel disease. J Intern Med 263(6): 591-596.

34. Qi YC, Xiao XJ, Duan RS, Yue YH, Zhang XL, et al. (2014) Effect of acupuncture on inflammatory cytokines expression of spastic cerebral palsy rats. Asian Pac J Trop Med 7(6): 492-495.

35. Li J, Li J, Chen R, Cai G (2015) Targeting NF- $\kappa$ B and TNF- $\alpha$ activation by electroacupuncture to suppress collagen-induced rheumatoid arthritis in model rats. Altern Ther Health Med 21(4): 26-34.

36. Szarka LA, Camilleri M (2010) Stomach dysfunction in diabetes mellitus: emerging technology and pharmacology. J Diabetes Sci Technol 4(1): 180-189.

ISSN: 2574-1241

DOI: 10.26717/BJSTR.2018.11.002153

Aline French de Lima Vitorino. Biomed J Sci \& Tech Res

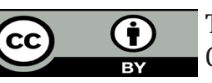

This work is licensed under Creative Commons Attribution 4.0 License

Submission Link: https://biomedres.us/submit-manuscript.php
37. Bueno L, Ferre JP, Ruckebusch M, Genton M, Pascaud X (1981) Continuous electrical and mechanical activity recording in the gut of the conscious rat. J Pharmacol Methods 6(2): 129-136.

38. Enck P, Wienbeck M (1989) Repeated noninvasive measurement of gastrointestinal transit in rats. Physiol Behav 46(4): 633-637.

39. Meunier LD (2006) Selection, acclimation, training, and preparation of dogs for the research setting. ILAR J 47(4): 326-347.

40. Tatewaki M, Harris M, Uemura K, Ueno T, Hoshino E, et al. (2003) Dual effects of acupuncture on gastric motility in conscious rats. Am J Physiol Regul Integr Comp Physiol 285(4): 862-872.

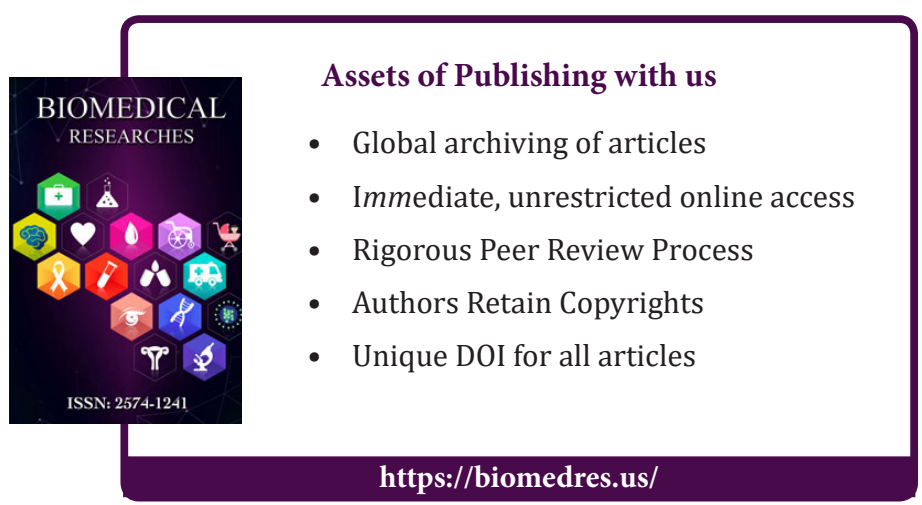

